requfsted him to collect as much of his saliva as he could, and he sent me about two ounces. This was evaporated, and left no yellow stain; the residue was treated with boilir $g$ alcohol, which was filtered and mixed with ether in a st pered bottle. In a few days a fine precipitate had formed, which, when removed and dissolved in water, afforded the characteristic colour of the biliary galts with sulphuric acid and sugar.

Now, if we are to look upon jaundice as consisting in the presence of the hepatic secretion in the blood, this was a case of jaundice without the presence of the jellow colour usually supposed to be characteristic of it. What is difficult of explanation is that the biliary salts must have been prest nt in the circulation for many months without any deterioration of the general health. Weare told that these salts, when injected into the blood, produce jaundice, hæmorrhage, and slowness of the pulse, but there were no such symptoms in the gentleman in question.

The detection of the biliary salts in the above case seems to throw light upon those cases of "biliousness" in which bitt erness of taste in the early morning is a prominent symptom. But you may fairly object that the presence of either the biliary pigment or of the taurocholate of soda in the circulation is insufficient to explain many of the symptoms from which a bilious patient suffers, for slight jaundice may be present for a considerable time without any great deterioration of the general health. You must remember, however, that under normal conditions the biliary salts are reabsorbed and decomposed in the circula. tion, and we may, therefore, suppose that in most bilious persons any excessive secretion of taurocholate of soda by the liver will be followed by an increased production of secondary sulphur componnds in the blood. Now, I am led hy various experiments to believe that most of the sym toms recognised as arising from an excessive secretion or undue retention of bile in the organism are the result of an injurious action produced by these secondary sulphur compounds on the tissues. This subject, although most interesting, is far too wide to be discussed in the present lectuse, but I hope on a future occasion to bring it under your notice.

\section{CASE OF PARALYSIS OF THE ABDUCTORS OF THE VOCAL CORDS.}

Being an extract from a Clinical Lecture on Paralytic Affections of the Laryno, delivered at the Royal Infirmary, Liverpool,

$$
\text { By T. R. G L Y N N, M.B. LoN D., }
$$

Gentumen, - I must now direct your attention to an example of a very uncommon affection of the laryngeal muscles-where the paralysis is confined to the abductors.

W. J-, aged thirty-six, married, a blacksmith, was admitted into No. 11 ward on January 16th, 1877. The moment he entered the out-patient room he excited your attention from the extraordinary noise he made; every inspiration was accompanied by a crowing sound similar to that made by a cuckoo clock or a child with croup, but much louder than either. On walking upstairs the exertion increased the dyspnoea from which he suffered, and exaggerated the noise into a discordant howl, which was heard throngh the neighbouring passages and wards, to the astonishment of nurses and patients. He told us (I now avail myself of the accurate notes taken by Mr. Hugh Williams) that " be had generally enjoyed good health, and was tem. perate in his habits. Never had any affection of the throat before. Had recently been laid up in the Northern Hospital suffering from an abscess and sinus in the right thigh, in consequence of an injury. There is no history of syphilis. On Christmas day he walked several miles near the sea; it was foggy, cold, and damp, and he felt chilled. In the afternoon or evening of that day he noticed a pricking sensation in the fauces, as if a pin were sticking there. A cough came on, and three or four days afterwards he found that he made a noise when be drew his breath; bis roice became weak, and his breathing difficult. He gradually grew worse, and for the last few nights has been unable to lie down.

"He is a light-complexioned, thin man. There is a sinus in the right thigh, and an eczematous rash on the right shoulder. His face is flushed. Respiration 26 ; each inspiration is accompanied with a loud cooing sound. The inepirations are laboured, and attended with sinking of the suprasternal, clavicular, and intercostal spaces. The expirations are more easy"; but they, too, were prolonged, there being little or no respiratory pause. "He has a noisy cough, and expectorates frothy mucus. Foice boarse and weak, but preserved; pulse 78, full and hard; tongue clean; appetite good ; urine normal."

Physical examination of lungs and heart disclosed nothing of moment.

"Examination of larynx. - The mucous membrane of the fauces appears to be redder than natural, and looks glossy. With the laryngoscope this appearance is found to extend into the larpnx. There is a small ulcer on the tongue, close to the middle glosso-epiglottidean fold. The glottis is narrowed to a mere triangular slit, the cords being closely approximated; they do not separate during inspiration." Subsequent examinations confirmed these appearances, and disclosed the fact that the cords actually approached each other still more closely during inspiration, when they distinctly vibrated, producing the noise described; and sepas rated during expiration.

"Jan. 17th.-He tried to sleep propped up in bed, and dozed a little; but he made so much noise when asleep that the patients would not permit him to close his eyes. As it wrs, no one could sleep near him or in the adjoining wards. When asleep, or when he lies down, his face becomes purple. His cough is very troublesome; he has expectorated a large quantity of frothy mucus. Ordered 'common diet'; ice to suck; and a mixture containing a quarter of a grain of extract of belladonna and twenty grains of bromide of potassium, three times a day. Fomentations to the neck.

" 18 th.-Had a much better night; did not make nearly so much noise; breathing much more quiet; inspiration only accompanied with a slight stridulous sound. In other respects be is much the same. Ordered iodide of potassium, ten grains, three times a day."

On the 19 th he was again as bad as ever. The patience of all in the ward was exbausted, being especially tried at night. He had not been allowed to sleep, his neighbours shouting at him or pinching him whenever he tried to doze. I could not shut the man up by himself, as we have here, as you know, no means whatever of isolating patients. I applied a laryngeal electrode over the crico-arytenoide: postici, using the interrupted current, without decided allo. viation, and then determined that tracheotomy should be performed. The operation was successfully carried out by Mr. Davison. The man was immediately relieved, and did very well, and peace was restored to the ward. On the 24 th a vulcanite tube was substituted for the metal one, much to his comfort.

" 25th.-Can speak (with tube closed, of course) much clearer than he could, but finds it diffcult to breathe through the glottis. Respiration 22; pulse 78.

of 26th.-Voice still stronger, and breathes through the glottis with very little noise. Allowed to get up. The mirror showed that some power of abduction was returning; the cords were continually being abducted and adducted, but only to a limited extent."

Soon after this he was furnished with a cork, which he kept in the tube by day, but took out when he went to bed in order that he might sleep without making a noise. When be did sleep with the cork in, those about him noticed that the sound be made had lost something of its old character, and was more like snoring. He remained in much the same condition for some time, and on March 1st I commenced using the interrupted current with Mackenzie's laryngeal electrode and necklet, after which he rapidly improved.

" March 9th.-The adductors are daily stimulated by the interrupted current. The glottis is much wider than formerly; the cords separate during inspiration now, and to the eighth of an inch.

" 13 th.-Discharged cured."

In considering the nature of this affection, you must bear in mind that every movement of the glottis, whether af 
contraction or dilatation, as well as every dainty adjustment for vocalisation, depends on muscular action. The arytenoid cartilages are delicately poised, and supported by the tonic contraction of the muscles about them; here muscular energy is not, as it often is, antagonised by elastic force; in the condition of equilibrium the cords are maintained apart; with every inspiration their equilibrium is temporarily disturbed by the action of the crico-arytenoidei postici, the cords being still further separated and the glottis widened for the entrance of air. These muscles are the sole dilators of the glottis. The entrance of air into the lungs and the passage of air through the trachea are equally dependent on muscular energy. The crico-arytenoidei postici may thus be regarded as muscles of inspiration. When we sow that the glottis was never thoroughly open, the inference necessarily was, either that these muscles were paralysed, or that their antagonists by violent action overpowered them. From the absence of anything paroxysmal about the affection, and from the persistence of the symptoms, I believe that the former explanation is the correct one.

I have aid we noticed that during inspiration the cords, instead of separating, actually approached each other more closely, that the narrow glottis was further narrowed, while during expiration the cords were separated and the chink widened. The explanation of this is, I think, to be found in the difference in the form of the upper and lower surfaces of the vocal cords. If you look at these casts (see fig.) of the interior of the healthy larynx, you will observe

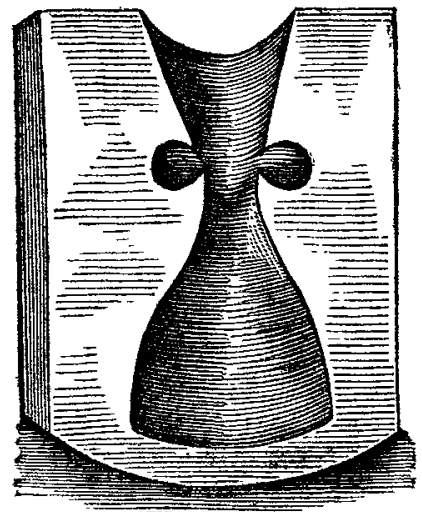

that the upper surface is flat or hollow, the lower oblique, therefore the inspiratory and expiratory blasts, when the cords are close together, act very differently upon them. While the inspiratory will drive them more closely together, the expiratory will separate them. This conformation of the cords leads to an aggravation of the dyspnoa in several affections of the glottis.

Oar patient could not, when at his worst, inspire or even expire without producing a noise, becanse his cords wer stretched and adducted in the position for phonation. His roice was weak and hoarse, probably from the absence of that support the arytenoid cartilages derive from the posterior crico-arytenoid muscles.

Ziemssen, in the "Cyclopædia of Medicine" (vol.vii., p. 959), gives particulars of nine cases of paralysis of the abductors of the cords. Eight he has collected from various sources, and one he observed himself. In these there was the same inspiratory dyspncea and stridor, the voice was in some cases weakened, but never lost. The symptoms were dereloped slowly and were persistent. One case was complicated with paresis of the soft palate, and in this there was some difficulty experienced in swallowing, but in none of the otbers. I need not dwell further on the symptoms of this affection.

I have reason to hope that my patient will reoover completely. The man has now (June 20 th) been without the tube since March; the orifice in the trachea has closed, his breathing is easp and noiseless, and his voice natural.

On turning to Ziemssen's cases, we find that perfect re. covery is uncommon. Out of the nine cases, one was completely cured, one improved sufficiently to breathe without the cannula, two continued to wear the cannula, four died, and one was lost sight of.

In the treatment of this affection stimulation of the affected muscles by the induced or constant currents is of the first importance. Situated at the back of the cricoid eartilage and lying immediately beneath the mucous mem- brane, these muscles are easily reached by an electrode passed into the pharynx. Tracheotomy must be resorted to if the symptoms become urgent; it was necessary in six of the cases before referred to.

I believe that the paralysis was due in my case to a morbid condition of the muscles themselves, and probably inflammatory and not dependent on any nerve lesion. There is the history of exposure to cold and of pain in the throat, the catarrhal condition of the mucous membrane of the throat, with the presence of an ulcer. This hypothesis seems also more in accordance with the facts observed than one which must invoke the existence of symmetrical and limited lesions of both recurrents.

\section{ON THE USE OF THE ASPIRATOR IN HEPATIC ABSCESS.}

BT E. H. CONDON, M.D., BURGEOY-MAJOR, ARMY MRDICAT DEPARTMHMY. (Conctuded from page 275.)

CaSE 7.-Private Wm. S-, 1st Batt. 21st Fusiliers, aged twenty-eight years; service nine years. Admitted into the General Hospital, Madras, on the 28th July, 1874, suffering from a severe attack of hepatitis. Tongue furred; bowels costive; pulse quick and full; skin hot and dry; respiration hurried; liver much enlarged, very painful and tender. He was treated on the usual principles, and his side leeched and fomented, \&c. The general pyrexia \&e. were relieved, but the pain, tenderness, and enlargement of the liver remained. The temperature was very high, usually $103^{\circ} \mathrm{F}$., but ranging between $99^{\circ}$ and $103^{\circ}$.

On the morning of August 9th his liver was explored in two places in the most prominent part of the right lobe. The needle came out perfectly dry, and the aspirator failed to draw even a little blood or moisture into it. In a few hours he was almost free from pain in his side, and the next morning (August 10th) the enlargement of his liver was found greatly reduced, the pain and tenderness almost gone, so that his side was percussed and manipulated freely without causing him the slightest inconvenience. His temperature was normal, and his respiration 24.

From this time he steadily improved, all enlargement of the liver disappeared, and he was pronounced convalescent on the 20 th of the same month.

He was under my observation in the regiment for about six months afterwards, and continued perfectly well, and always attributed his recovery to the operation.

CAsa 8.-Corporal G. W. R-, R.A., aged twenty-three years; service three years. Admitted into the General Hospital, Madras, on the 21st August, 1874, suffering from a mild attack of hepatitis. There was only slight enlargement of the liver, but there was severe pain, which was confined to a small space between the cartilages of the eighth and ninth ribs. There was, however, extensive tenderness radiating from that spot, chiefly downward and to the left side; his temperature was usually $100^{\circ}$ every evening, and only once it was $102^{\circ}$; his pulse ranged between 90 and 109, and his respiration between 24 and 36. His tongue was furred and his face sallow, with an anxious expression of countenance. He had no distinct rigors, but he occasionally perspired very severely.

He was treated with chloride of ammonium, Dover's powders, \&c., and hot fomentations locally.

There was little change in his condition until the 8th of September, when the fine needle of the aspirator was passed into the liver between the cartilages of the eighth and ninth ribs to the depth of two inches, and about an ounce of the usual brownish pus drawn off. The operation relieved his pain, and he slept well that night for the first time since he was admitted into hospital. His temperature fell to normal, and never rose bigher than $988^{\circ}$ af terwards.

On the 15th September two ounces of thick pus were drawn off, the last balf-ounce of which was bloody; the abscess was evidently emptied. 